\title{
De las visiones coloniales a las políticas postcoloniales: el caso de los San de Namibia
}

From colonial views to postcolonial politics. The case of the San in Namibia

\section{Eduard Gargallo}

\section{(2) OpenEdition \\ 1 Journals}

Edição electrónica

URL: http://journals.openedition.org/aa/1394

DOI: $10.4000 / a a .1394$

ISSN: 2357-738X

Editora

Programa de Pós-Graduação em Antropologia Social (UnB)

\section{Edição impressa}

Data de publição: 1 dezembro 2015

Paginação: 51-76

ISSN: 0102-4302

\section{Refêrencia eletrónica}

Eduard Gargallo, «De las visiones coloniales a las políticas postcoloniales: el caso de los San de Namibia», Anuário Antropológico [Online], v.40 n.2 | 2015, posto online no dia 01 junho 2018, consultado o 28 abril 2021. URL: http://journals.openedition.org/aa/1394 ; DOI: https://doi.org/ 10.4000/aa. 1394

\section{(c) (i) (9)}

Anuário Antropológico is licensed under a Creative Commons Atribuição-Uso Não-Comercial-Proibição de realização de Obras Derivadas 4.0 International. 


\section{De las visiones coloniales a las políticas postcoloniales: el caso de los San de Namibia*}

Eduard Gargallo

ISCTE-IUL

\section{Introducción}

Cuando hablamos de la importancia de las clasificaciones coloniales de determinadas culturas o grupos de población en África, y de su permanencia en el período postcolonial, no hay duda que las comunidades San (o Bosquimanas) ${ }^{1}$ del África Austral pueden ser consideradas como unas de las más intensamente - y negativamente - afectadas. En el caso concreto de Namibia, la visión que los europeos desarrollaron de los San afectó su tratamiento bajo la administración colonial y desembocó en una pérdida gradual de derechos, de acceso a los recursos y, como analizaremos especialmente en este artículo, de control sobre sus tierras históricas. El acceso de Namibia a la independencia en 1990 no ha modificado sustancialmente muchas de las ideas existentes sobre los San, ni ha supuesto un cambio de políticas que haya corregido la marginalización extrema de estas poblaciones.

Es importante resaltar, sin embargo, algunos aspectos que añaden complejidad y ambigüedad a la descripción esbozada en el párrafo anterior. En primer lugar el discurso "colonial" sobre los San, en Namibia, no fue homogéneo ni fijo a lo largo del tiempo. En segundo lugar, la visión marginalizante y despectiva sobre los Bosquimanos no ha sido nunca únicamente producida por los europeos. Los africanos, ya durante el período pre-colonial, y especialmente desde la colonización y en la post-independencia, han desarrollado imágenes a menudo negativas de sus vecinos San, o apropiándose de partes del discurso europeo, o elaborando uno propio, o combinándolos. Finalmente, aunque en este artículo no podamos analizarlo, las mismas comunidades San han sido agentes en la formulación de su propia identidad, y han hecho uso de elementos étnicos y culturales para conseguir sus propios fines (Robins, 2000).

Hemos decidido dedicar una atención especial a la cuestión de la tierra porque, a pesar de no ser la única que refleja la marginación de las comunidades San, ocupa un lugar central en sus reivindicaciones, su identidad y su cultura. Namibia es uno de los dos países con mayor población San (junto con la vecina Botswana) y, como veremos, ha desarrollado una política menos agresiva hacia las reivindicaciones 
bosquimanas, pero también se ha negado a seguir el ejemplo sudafricano de reconocimiento del derecho de reclamación de las tierras ancestrales.

Nuestro trabajo se basa en el uso de fuentes bibliográficas, informes y documentos oficiales, y trabajo de campo a lo largo de diversas estancias en Namibia, primero entre 2007 y 2009, y posteriormente en 2012 y 2014. Durante estas estancias se han realizado entrevistas a oficiales del gobierno, ONGs, organizaciones de defensa de los derechos de los San y a poblaciones San tanto en granjas de reasentamiento como en tierras comunales.

\section{Las comunidades San en la Namibia precolonial}

Grupos de poblaciones San ocuparon amplias áreas del actual Estado de Namibia a lo largo de siglos. Dedicados esencialmente a la caza y la recolección, esto no les impedía desarrollar otras actividades económicas de forma puntual, como el comercio con comunidades bantúes y Khoikhoi vecinas, o la adopción de la agricultura o la ganadería a pequeña escala en lugares y momentos específicos. ${ }^{2}$ Los San no conocían la "propiedad" de la tierra en el sentido europeo del término, pero sí existían territorios determinados a los que un grupo o banda tenía acceso prioritario y era necesario pedir permiso al grupo que los controlaba antes de cazar o recolectar en ellos. Los derechos de uso sobre uno o diversos territorios se obtenían por nacimiento, por matrimonio o por residencia. En el Kalahari, se organizaban en bandas de unos 20 a 50 individuos, ligados por parentesco o lazos de dependencia. La caza-recolección comunal y el intercambio dentro de una banda, y entre ellas, aseguraban un elevado grado de igualdad socioeconómica en muchas de las poblaciones San. La ausencia de líderes políticos significativos y la toma de decisiones basada en el consenso entre miembros de las bandas reforzaban esta tendencia igualitaria (Barnard, 1992; Guenther, 1999; Smith et al., 2000).

Las comunidades San no quedaron, de todas formas, al margen de los cambios socioeconómicos y políticos que afectaron a Namibia a lo largo del siglo XIX, impulsados tanto por la llegada de comerciantes y viajeros europeos a la región, como por las migraciones de diversos grupos africanos: los Oorlam-Nama ${ }^{3}$ provenientes de la Colonia del Cabo, organizados en comunidades diversas bajo líderes político-militares, o los Herero desplazados por los Oorlam y en busca de pastos para su ganado. Si bien con anterioridad los San ya habían tenido contactos comerciales y laborales con sus vecinos, su participación en la economía regional se intensificó y los intercambios de productos se hicieron más frecuentes (Dieckmann, 2007; Gordon, 1992; Rousset, 2003).

Asimismo, en las décadas centrales del siglo XIX se puso en marcha un proceso de pérdida progresiva del control de determinados territorios por parte 
de los San. Los pastores Herero se habían desplazado más al norte desde el centro del país, huyendo de los ataques de los Oorlam-Nama, y cuando, más tarde, pudieron recuperar su fuerza y sus rebaños, continuaron su expansión. Zonas que actualmente se consideran parte de "Hereroland", como Okamahuti, Otjituo, o las Otjitjika Mountains, hasta el siglo XIX habían sido habitadas por los San (Gordon, 1992). En el Omaheke ciertos grupos de Ju/'hoan San también se convirtieron en siervos o clientes de los Herero o los Tswana, mientras otros pudieron mantener su autonomía (Sylvain, 1999).

\section{El primer impacto colonial}

Alemania declaró los territorios de la actual Namibia, entonces llamada África del Sudoeste, como protectorado en 1884 y en los años siguientes extendió un control más o menos firme sobre amplias áreas del país, incluyendo algunas donde residían comunidades San.

Desde el siglo XVII, los europeos asentados en el Sur de África habían desarrollado una visión extremadamente negativa de las poblaciones Khoisan, entonces conocidos como Hotentotes y Bosquimanos. Los holandeses, afincados en el Cabo desde 1652, consideraban a estas comunidades, especialmente a los llamados Bosjeman, como escasamente humanos, sin una lengua propiamente dicha — se decía que se comunicaban a través de "sonidos" únicamente —, incapaces de establecer reinos u organizaciones políticas mínimamente sofisticadas, y carentes de jefes (Chidester, 1996). Ni siquiera se les atribuían creencias espirituales o religiosas (Penn, 1996; Elphick, 1977). Esta visión se radicalizó a lo largo del siglo XVIII y se plasmó en una aceleración de las matanzas de San. Las campañas de exterminio se justificaban por la ausencia de líderes con los que negociar, por la escasa "docilidad" de los hombres San y la supuesta imposibilidad de adaptarlos a la vida de sirvientes. Con frecuencia, en un ambiente de frontera en expansión, los San eran considerados como inferiores incluso a los menospreciados "Hotentotes", como simple "vermin" (alimañas) (Penn, 2005).

Los estereotipos sobre los San se prolongaron e intensificaron durante el siglo XIX, a medida que los británicos del Cabo, primero, y viajeros de múltiples nacionalidades, después, entraban en contacto con ellos en las diferentes regiones del África Austral. Proliferaron en esa época las capturas de hombres y mujeres San para mostrarlos en circos y exhibiciones en Europa y los Estados Unidos. Estas actividades iban acompañadas a menudo por explicaciones "científicas" que repetían las ideas habituales: los San eran caracterizados como salvajes, extremadamente primitivos, carentes de lengua y religión, y se aseguraba que vivían en cuevas o bajo tierra (Gordon, 1997; Peacock, 1999). 
Con este bagaje cultural, los primeros oficiales y colonos alemanes se establecieron en el África del Sudoeste y se apropiaron de tierras anteriormente habitadas por los San, quienes fueron forzados a trabajar en las granjas europeas o murieron por la acción de patrullas militares. Algunos grupos huyeron a zonas más apartadas o respondieron atacando a los granjeros aislados (Dieckmann, 2007; Gordon, 1992).

En 1904, los Herero primero y los Nama poco después se alzaron en armas contra los alemanes, quienes respondieron con una campaña de exterminio contra las poblaciones implicadas en el alzamiento (Silvester \& Gewald, 2003). En este contexto de violencia extrema contra los africanos, los alemanes — que mantenían la visión racista y negativa de los San como salvajes primitivos que robaban ganado y tal vez habían colaborado en el alzamiento - intensificaron las patrullas contra comunidades Bosquimanas, así como las matanzas y la captura de individuos destinados al trabajo forzado. Esta política se mantuvo incluso años después del fin de la guerra con los Herero y Nama (Gordon, 1992).

\section{La administración sudafricana}

Durante la Primera Guerra Mundial, las tropas sudafricanas ocuparon África del Sudoeste y, en 1920, Sudáfrica recibió de la Sociedad de Naciones el mandato para administrar el territorio. En los primeros momentos de la ocupación sudafricana, el trato a los africanos mejoró sensiblemente. La administración suavizó algunas de las leyes alemanas, por ejemplo reduciendo los castigos físicos a los africanos (Werner, 1998). Pero en seguida quedó claro que los sudafricanos no pensaban rectificar las líneas básicas de la distribución de tierras y, en 1920, el gobierno de Pretoria anunció el inicio de un programa para impulsar la llegada de colonos sudafricanos, que debían instalarse en ranchos ganaderos distribuidos por las zonas centrales y meridionales de Namibia, incluidas algunas áreas ocupadas o utilizadas por los San (Adams, Werner \& Vale, 1990; Silvester, 1993).

Con respecto a los San, los sudafricanos llegaron con una historia - como hemos visto - de desprecio y hostilidad hacia ellos. El informe elaborado en 1918 para detallar las atrocidades de la administración alemana en el territorio, incluía afirmaciones como que "el modo de vida de los Bosquimanos salvajes puede ser descrito como escasamente superior al del león y el leopardo - sus rivales en la caza", o que "tienen sólo las más vagas ideas sobre la propiedad o las pertenencias" (Silvester \& Gewald, 2003: 236). Fourie, oficial médico del territorio, decía que los San no tenían "organización tribal” alguna, aunque ya hacía una razonable descripción del uso del territorio por las bandas bosquimanas (Hahn, Fourie \& Vedder, 1928). En la mentalidad del momento, los San eran a 
menudo infantilizados, equiparando su capacidad intelectual a la de los niños europeos (Sylvain, 1999).

Fue también en los años 20, sin embargo, cuando empezaron a aflorar visiones un poco menos agresivas de los San, pero que eran igualmente estereotipadas y paternalistas. La conocida como Denver African Expedition norteamericana de 1925 realizó películas y fotografías que reforzaban el deseo de mostrar a los San como no "contaminados" por el contacto con los europeos, y como "puros" representantes de una población salvaje y primitiva. Se escondía deliberadamente tanto su historia como, por ejemplo, el uso de ropa de estilo europeo (Gordon, 1997).

En un ámbito más práctico, los sudafricanos establecieron un sistema de Reservas de tierra limitadas para los africanos, donde podrían mantener su ganado, la propiedad de la tierra sería comunal y gestionada por los jefes tradicionales. Las Reservas también facilitarían el control administrativo de la población. En estos primeros ańos, no se preveía el establecimiento de ninguna Reserva destinada a los San (Adams, Werner \& Vale, 1990; Hinz, 1998; Kössler, 2005; Werner, 1998).

Durante los años de entre guerras, los San fueron perdiendo aún más terreno a medida que el número de granjeros blancos aumentaba. Los Hai//om fueron incorporados a la economía colonial, igual que durante la época alemana, con una mezcla de coerción, patrullas policiales y atracción por los salarios o productos europeos. La gran mayoría de ellos pasaron a trabajar en las granjas de los europeos de la zona, ya fuese como pastores, peones o sirvientes domésticos (Dieckmann, 2007; Gordon, 1992). La creación de Reservas y el aumento de población en las mismas también colaboraron al desplazamiento de comunidades San. Cuando se estableció la Reserva de Waterberg East, los Herero residentes y recién llegados fueron ocupando tierras anteriormente usadas por los San, que se vieron forzados a trabajar - aunque fuera temporalmente - como pastores o recogedores de leña para los Herero a cambio de comida, tabaco y alguna cabra o vaca ocasional (Gordon, 1992).

Los Herero compartían, en general, la visión que tenían los granjeros blancos sobre los San: creían que era necesario usar la fuerza para obligarles a trabajar, y les calificaban frecuentemente de "serpientes" o "perros salvajes". Los europeos, por su parte, defendían la práctica de no pagar un salario monetario a los San, o que el sueldo fuera mínimo, con la teoría de que ya complementaban sus ingresos con la caza y la recolección, y además eran estúpidos y no "entendían" el valor del dinero (Suzman, 2000; Sylvain, 1999). En Owamboland, el crecimiento demográfico y la llegada de poblaciones Owambo que huían de la Angola portuguesa provocaron la expansión de las comunidades Owambo hacia regiones más remotas, tradicionalmente consideradas poco valiosas para la 
agricultura y la ganadería, y donde los San habían cazado y recolectado. Ahora todas las áreas se volvían necesarias, con lo que se redujo el número de animales salvajes y se ocuparon casi todos los puntos de agua. Los Owambo recién llegados consideraban que estos puntos estaban "libres", y cuando realizaban mejoras en los pozos o charcas, se los apropiaban (Kreike, 1996).

\section{La última fase del gobierno colonial}

En los años 1950 y 1960, el aumento del número de granjeros y el levantamiento acelerado de vallados redujeron aún más las posibilidades de los San para moverse por el territorio o cazar y recolectar en él; entonces, un número creciente entró al servicio de granjeros blancos (Dieckmann, 2007; Gordon, 1992). Además, los San fueron expulsados gradualmente de los Parques Nacionales y otros espacios protegidos (Dieckmann, 2007; Orth, 2003). Este período de pérdida creciente de tierras, sin embargo, coincidió con la expansión de visiones más favorables de los San entre los europeos de la región Austral. De hecho, antropólogos alemanes de principios de siglo XX ya habían alertado de la necesidad de salvar "reliquias" de los Bosquimanos para su estudio, aunque fuese como muestra de una humanidad primitiva, incluso próxima a los simios (Gordon, 2003).

En Sudáfrica, antropólogos y otros científicos, en los años 1920, mostraron su interés por las poblaciones San y por preservar sus "reliquias" en museos. En los años posteriores a la Segunda Guerra Mundial, se intensificaron los mensajes que retrataban a los San como los únicos africanos "tradicionales" que quedaban, pero que estaban "desapareciendo" con rapidez. La visión más benévola sobre los San no escondía la idea de que los africanos habían históricamente desplazado a los San de sus territorios, justificando la ocupación de tierras africanas por parte de los europeos posteriormente (Gordon, 2003). La idea más repetida era la de crear una reserva, para que la ciencia los estudiase y para evitar su extinción (Gordon, 1992). Las descripciones de los San como representantes del "buen salvaje", supervivientes de los grupos humanos prehistóricos y con lazos "especiales" con la naturaleza, se fueron extendiendo en los años 1950 y 1960 entre antropólogos y algunos populares escritores blancos sudafricanos (Marshall Thomas, 1959; Van der Post, 1958).

Entre 1961 y 1964, la conocida como Odendaal Commission fue establecida por el gobierno sudafricano para analizar el sistema político y de distribución de tierras en África del Sudoeste, y recomendó extender al territorio el sistema de bantustans que ya se estaba poniendo en marcha en Sudáfrica. La Comisión y los ideólogos del régimen consideraban que cada grupo étnico africano tenía unas características culturales específicas y que se encontraban en un estado 
de desarrollo diferente. Por tanto, no podían integrarse ni entre ellos ni con los europeos en un único sistema de gobierno. Había que crear homelands o territorios en los que cada grupo tendría derechos exclusivos de residencia y unas autoridades propias autónomas (Kössler, 2005; Pisani, 2000).

La Comisión analizó el caso específico de los San, y se decidió que los únicos con derecho a un territorio propio serían los Ju/'hoan de la región de Nyae Nyae, que fueron considerados como los únicos "salvajes" y, por tanto, "auténticos". En un ejemplo especialmente crudo de los lazos entre la categorización colonial de las poblaciones y la práctica política, los Hai//om o los Khwe se consideraron como demasiado "occidentalizados" para disponer de una identidad "pura" y en consecuencia no se les reconoció derecho a tierras propias (Gordon, 1992).

Siguiendo las directrices del Plan Odendaal, a partir de 1968, las 17 Reservas africanas anteriores se agruparon en 7 homelands, y en 1976 se les añadió "Bushmanland" (Adams, Werner \& Vale, 1990; Hinz, 1998). A la vez, una nueva versión "positiva" de la cultura San se fue desarrollando entre el establishment sudafricano y, especialmente, dentro de la South African Defence Force. Según estos planteamientos, los San tenían unas características naturales de adaptación al medio natural, un oído más desarrollado, una gran habilidad de orientación en la sabana, una capacidad de resistencia que iba más allá de los humanos "normales", y esto les hacía susceptibles de ser reclutados por el ejército en su campaña de lucha contra las guerrillas nacionalistas (Gordon, 1992; Robins, 2000).

Los cambios en la distribución de tierras tras la aplicación del Plan Odendaal perjudicaron de nuevo a los San. Tierras que habían sido tradicionalmente usadas por los Bosquimanos en la región de Nyae Nyae fueron integradas en Hereroland y Tswanaland por el sur y en Kavango por el norte. El homeland de Bushmanland era así mucho menor de lo que habían sido los territorios San anteriormente. Bushmanland, además, era un homeland atípico puesto que, a diferencia de los demás, y de acuerdo con la visión colonial tradicional de los Bosquimanos, no tenía una autoridad tribal que lo administrase y era gobernado directamente por oficiales blancos (Botelle \& Rohde, 1995; Gordon, 1992).

En las tierras propiedad de los europeos continuó la incorporación de los San a los trabajos agrícolas. Según el censo de 1981, en las granjas del centro y del norte el 26\% de los trabajadores eran San, el segundo grupo más numeroso (Pankhurst, 1996). En las granjas, se mantenía el principio del baaskap: una relación empleado/ granjero que incluía altas dosis de paternalismo por parte del dueño hacia sus trabajadores (les ayudaba con problemas familiares, la escolarización de los hijos, etc) acompañadas por un fuerte autoritarismo, la exigencia de obediencia absoluta al granjero y el derecho de éste a "castigar" a sus trabajadores (Sylvain, 1999). 
En el caso concreto de los San, estos principios eran más agudos que para los demás empleados. Los San recibían menos salario que los demás, a veces únicamente comida y tabaco. Los granjeros les consideraban más débiles y menos fiables. Y les ofrecían menores raciones alimentarias, porque esperaban que buscasen el resto de su comida en la sabana (Gordon, 1992).

\section{La Namibia independiente}

Tras una larga y cruenta guerra de liberación (1966-1989), y unas complejas negociaciones entre la Swapo, el principal grupo nacionalista, Sudáfrica y diversos países occidentales y africanos, Namibia se convirtió en un Estado independiente en 1990. La Swapo ganó las elecciones y accedió al poder. Sin embargo, el fin del período colonial no supuso una mejora sustancial de la situación de los San o de su relación con el Estado pues, como veremos, la visión de los nuevos gobernantes sobre los San era poco favorable. La Swapo provenía de una tradición ideológica altamente centralizadora, influida por las teorías políticas marxistas, y deseaba romper con el pasado de división étnica del país desarrollada por los sudafricanos. Las reivindicaciones de tipo cultural e identitario eran especialmente mal recibidas (Graefe \& Peyroux, 1999; Keulder, 1998). Los homelands desaparecieron y se estableció una nueva división territorial en 13 regiones con fronteras demarcadas no por criterios étnicos sino en función de la geografía y la economía (Tötemeyer, 2000). La Swapo recortó también el poder de las Autoridades Tradicionales, que disfrutan de competencias en diversos ámbitos y tienen el derecho de atribuir tierra en primera instancia, pero están obligadas a colaborar con las políticas del Estado y deben ser reconocidas oficialmente por el Gobierno (Harring, 1998; Keulder, 2000). Como veremos con más detalle, esto afectó a los San principalmente en dos planos: al disponer de muy pocas tierras comunitarias reconocidas como propias, quedaron bajo la jurisdicción de Autoridades de otros grupos; y muchas comunidades San no disponían de Autoridades Tradicionales históricas, o el gobierno y los grupos étnicos vecinos no se las reconocían.

Actualmente, las diferentes comunidades San en el país comprenden a unas 38.000 personas, lo que supone casi un $2 \%$ de la población namibia total (WIMSA, 2007-2008). Los San se encuentran en la situación más precaria de todos los grupos étnicos namibios según todos los indicadores de desarrollo humano. Su esperanza de vida en 1998 era de 48,1 años, un 22\% menor a la media nacional de 61. Pese a un aumento muy considerable en la escolarización de los niños San desde la independencia, las tasas de abandono escolar a una edad temprana son muy elevadas, y su índice de alfabetización era del $23 \%$ frente al 66\% general (Suzman, 2001). Si bien Namibia se considera como un país de renta 
media, en 2007, el PNUD afirmaba que el Índice de Desarrollo Humano de los San namibios era de 0,359, parecido al de países como Sierra Leona o la República Centroafricana (WIMSA, 2007-2008).

\section{La cuestión de la tierra}

Las reformas agrarias en el África Austral están envueltas en cuestiones relacionadas con la etnicidad y los derechos de las comunidades llamadas "indígenas". De forma simplificada, el debate gira en torno a tres puntos: definir qué grupos pueden ser considerados "indígenas"; determinar si algunos grupos étnicos tienen que ser prioritarios a la hora de la redistribución de tierras, con base en su historia particular de despojo durante el período colonial; y decidir si la reforma debe centrarse en corregir las injusticias del colonialismo, retornando las tierras "ancestrales" a los grupos que las perdieron o si, por el contrario, es suficiente con el traspaso de tierras a los "africanos" en general, sin compensar a comunidades concretas con terrenos específicos. En los países de la región, las comunidades San, con el apoyo de los donantes, las ONGs y los movimientos internacionales de defensa de los pueblos indígenas han desarrollado campañas de defensa de sus derechos como los "primeros" habitantes de la zona, enfatizando la diferenciación étnica y los elementos culturales propios de los San. Esto ha sembrado las semillas de conflictos con los gobiernos que, desde las independencias, han desarrollado procesos de "construcción nacional" específicamente hostiles a las reivindicaciones étnicas (Hohmann, 2003).

Hay que indicar que Namibia ha mostrado una mayor voluntad de aceptación de la diversidad étnica que la vecina Botswana (Hohmann, 2003). Pero, aún así, las demandas de tierra por parte de grupos específicos que fueron expropiados durante el período colonial son vistas por el gobierno como demandas "tribales". Oficialmente, todos los namibios negros son "indígenas", y Namibia se ha mostrado reticente ante los acuerdos internacionales de defensa de los pueblos indígenas (Hoffmann, 2005; Suzman, 2001).

\section{El programa de Reasentamientos}

En 1991 las autoridades convocaron la National Conference on Land Reform and the Land Question, en la que participaron todos los sectores implicados en la gestión de la tierra. La Conferencia aprobó una serie de resoluciones que tenían un carácter meramente consultivo. Entre ellas cabe destacar que la reforma agraria en Namibia no debía basarse en la reclamación de las "tierras ancestrales" perdidas por cada comunidad durante el período colonial. Se consideraba que este tipo de demandas no tendrían éxito por la "complejidad de las reclamaciones 
superpuestas" sobre una misma tierra. Al mismo tiempo, sin embargo, se proponía que en las zonas comunales debían priorizarse los derechos de los San a una tierra propia (MLR, 2005a). La National Resettlement Policy, aprobada en 1997, siguió en buena parte estas directrices y, por un lado consideraba beneficiarios potenciales del reasentamiento en tierras comerciales adquiridas por el gobierno a todos los namibios negros, mientras por otro lado indicaba como uno de los grupos prioritarios a la comunidad San, que era el único grupo étnico señalado de forma específica en las leyes de reforma agraria (MLRR, 1997).

En Namibia, se conoce como Reasentamientos a las granjas propiedad de blancos que el gobierno compra para redistribuir entre la población negroafricana. Pueden ser de dos tipos: reasentamientos individuales, en los que una granja se divide en parcelas individuales que se entregan a un beneficiario; o reasentamientos de grupos en los que la granja se explota de forma comunal por todos los beneficiarios (MLRR, 1997).

El Director de Reasentamientos repetía que se debían reasentar a personas de diversas regiones mezcladas porque "no queremos la política del apartheid otra vez” (S. H. Kandji comunicación personal, 28 mayo 2008). No existe un desglose por grupo lingüístico de los reasentados, y es difícil hacer una estimación del porcentaje que cada comunidad aporta a los Reasentamientos. Aunque, como hemos visto, los San son reconocidos oficialmente como uno de los grupos prioritarios a la hora de seleccionar a los beneficiarios del proceso de reasentamiento, esto normalmente no se ha llevado a la práctica. La gran mayoría de $S a n$ reasentados se encuentran en Reasentamientos de grupos y en condiciones muy precarias.

La situación en muchos de los Reasentamientos con poblaciones San significativas es bastante complicada. En Drimiopsis, en la región de Gobabis, algo más de la mitad de los residentes son San (DRFN, 2006a; Mosotho \& Tsiu, 2008). Un estudio del Namibia Institute for Democracy en 2008 calificaba la producción agrícola en Drimiopsis de "pobre" (Mosotho \& Tsiu, 2008:18). En la granja Skoonheid, en el mismo distrito de Gobabis, 73 familias se instalaron en el Reasentamiento, la mayoría San que habían perdido su trabajo en granjas de la zona. El gobierno construyó casas, pozos de agua nuevos e instaló un sistema moderno de riego. Parte de los pastos son usados por Herero vecinos para su ganado (DRFN, 2006b; Tapia, 2005). La Desert Research Foundation of Namibia en 2006 identificó a un 44,8\% de familias como "pobres", con ingresos esporádicos o inexistentes, y siempre insuficientes (DRFN, 2006b).

Muchos Coordinadores del MLR en los Reasentamientos son de etnias diferentes a los San, lo cual ha provocado tensiones. Por ejemplo, hubo muchas 
quejas contra una Coordinadora del MLR en Skoonheid, de etnia Tswana, que al parecer usaba a los beneficiarios como trabajadores domésticos (Suzman, 2000; Tapia, 2005). El Coordinador en 2008 también resaltaba la existencia de conflictos entre los diferentes grupos de beneficiarios. Según él, las demás comunidades en el Reasentamiento intentan "minar" los esfuerzos de los San, y las comunidades vecinas les "engañan” diciéndoles que deberían trabajar sólo por dinero en metálico y que si trabajan en los campos sin cobrar están trabajando a cambio de "nada" (Mr. Sijaja, comunicación personal, 10 junio 2008).

\section{Trabajo en las granjas comerciales}

Una parte significativa de las poblaciones San se encuentra en las granjas comerciales que aún son mayoritariamente propiedad de namibios blancos. Allí desarrollan diversos trabajos agrícolas, ganaderos o domésticos para los propietarios. A mediados de los años 1990 había unos 2.313 trabajadores San en estas granjas, lo que suponía el 6,4\% del total de trabajadores agrícolas del país (Suzman, 2001). Si calculamos una media de 6 personas por familia para cada trabajador, más de 13.800 San — o sea más de un tercio de las comunidades Bosquimanas — residían en granjas.

Una serie de cambios legislativos y la presencia de Inspectores de Trabajo en las granjas, han hecho que, desde la independencia, las condiciones laborales hayan mejorado, la violencia física contra los trabajadores haya disminuido claramente, y las relaciones laborales se hayan vuelto más "empresariales" (Sylvain, 1999; Werner, 2004). Pero en muchos casos cada granja sigue funcionando de hecho de forma autónoma y con condiciones variables en función del carácter del propietario, oscilando entre las más mecanizadas, modernas, donde se pagan mejores salarios y las raciones son una parte menor de la remuneración, hasta las más arcaicas, donde los salarios son inferiores y las raciones son un porcentaje mayor del pago (Sylvain, 1999).

La situación de los trabajadores San a menudo mantiene elementos del viejo sistema paternalista/autoritario en la relación granjero/empleado: normalmente el salario está formado en parte por raciones alimentarias, muchas veces insuficientes o monótonas; o los trabajadores son "heredados" junto con la granja por el hijo del granjero, o "recibidos" por el nuevo granjero en caso de que la propiedad se haya vendido. También ha habido casos de trabajo infantil, que casi nunca es remunerado. Algunos granjeros siguen justificando estas condiciones con el argumento de que los trabajadores San son supuestamente poco "fiables"; que los Owambo y otros grupos son más "profesionales" y más educados; que los Herero conocen mejor el ganado; o que las mujeres San son menos inteligentes 
y trabajadoras que las empleadas de otros grupos. Al mismo tiempo también se mantienen formas de paternalismo que incluyen la ayuda por parte de los propietarios para la escolarización de los hijos de sus empleados o contribuciones para los gastos médicos (Sylvain, 1999).

\section{Las Tierras Comunales}

La Constitución namibia afirma que las tierras de los antiguos homelands, ahora llamadas Áreas Comunales (ACs), son propiedad del Estado (Harring, 1998), aunque la National Land Policy (NLP) de 1998 ya advertía que las Autoridades Tradicionales tenían derecho a autorizar o excluir del acceso a tierras a personas de fuera de las comunidades correspondientes. Según la Communal Land Reform Act de 2002, las Autoridades Tradicionales veían reconocido su derecho a atribuir las tierras en primera instancia, siempre que sean para dedicarlas a cultivos o para uso residencial. Las tierras de pasto siguen estando bajo un régimen de explotación comunal y no pueden ser valladas (MLR, 2005a, 2005b; MLRR, 1998; Odendaal \& Tjiramba, 2005).

La precariedad de la situación de los San viene marcada, en primer lugar, porque no disponen prácticamente de tierras comunales propias, puesto que la única zona que los sudafricanos les reservaron era Bushmanland, con una capacidad de mantenimiento de población muy limitada. Se calcula que del total de población San de Namibia sólo el 15\% reside en la antigua Bushmanland (Harring \& Odendaal, 2006). En las demás áreas se ven obligados a solicitar permiso para usar la tierra a las Autoridades Tradicionales de otros grupos étnicos, cosa que resulta complicada porque en casi todas las ACs los San no llegan a ser el $10 \%$ de la población, por los estereotipos a que se ven sometidos por parte de las demás comunidades, y por la escasez general de tierra libre en estas zonas.

La visión que los restantes grupos étnicos tienen de los San continúa siendo en general negativa. Ya en la época alemana se han documentado casos como el de un juicio sobre tierras de los $\mathrm{Hai} / / \mathrm{om}$, en el que todos los implicados (Owambo, Herero y administración alemana) reconocen que los Hai//om fueron los primeros ocupantes de la zona, pero todos coinciden en negar ningún valor a esa presencia cuando se trata de dirimir la "propiedad" del territorio (Widlok, 2003). Las tradiciones orales de los Kavango, por su parte, reconocen que cuando sus ancestros llegaron a la región se encontraron con poblaciones San, pero eran gente "de la sabana”, sólo acudían al río Kavango ocasionalmente, no permanecían allí, ni tenían cultivos. Por lo tanto, no tienen derecho a poseer aquella tierra (McKittrick, 2008). 
La discriminación abarca muchos ámbitos. Está extendida la creencia de que los San nunca habían tenido Autoridades Tradicionales, aunque se sabe que, en ciertos casos, como en el Omaheke en el siglo XIX, la mayor presencia y los numerosos ataques de los Herero y Nama indujeron un proceso de militarización de los San y surgieron ciertos líderes políticos. Cuando en los años 1990 unos Mbukushu fueron acusados de intentar violar a una chica Khwe, una de las cosas que dijeron a los Khwe fue que ellos nunca tendrían jefes (Sylvain, 1999).

En la zona de Donkerbos/Sonneblom, con la mediación de WIMSA, los San obtuvieron la cesión de 13.000 hectáreas de tierra por parte de los jefes Herero, pero los Herero de los alrededores han expresado en ocasiones el deseo de recuperar "sus" tierras, y han enviado su ganado a pastar en el Reasentamiento (Sylvain, 1999). Casos como éste son raros (Suzman, 2001). Más frecuentes son situaciones como la de la región de Oshikoto, donde las Autoridades Tradicionales han seguido permitiendo el vallado de parcelas de tierra por parte de Owambos ricos y esto ha dejado a los San con cada vez menos acceso a tierras y a puntos de agua (Widlok, 2003). El acceso al agua en Áreas Comunales, sobre todo en las áreas centrales del país, con un clima semi-árido y muy pocas fuentes de agua permanentes, es un problema generalizado. Allí donde existen pozos comunitarios, el grupo mayoritario exige a los San que paguen una tarifa por su uso y, si no pueden pagarla, les impiden utilizar el pozo (WIMSA, comunicación personal, 23 febrero 2009).

En vista de todo esto, muchos San, en tierras comunales, deben dedicarse a trabajos esporádicos para campesinos de otros grupos que les ofrecen simplemente comida y alojamiento, o tal vez dinero para la escuela de sus hijos. Las condiciones laborales suelen ser peores que en las granjas comerciales, pero a veces los San prefieren trabajar para campesinos comunales al sentirse socialmente más cercanos a ellos que a los granjeros blancos. Muy pocos San han conseguido acumular ganado a causa de su pobreza, de la falta de derechos sobre zonas de pastos y de los robos que sufren con frecuencia. Sus huertos acostumbran ser pequeños y con producciones muy bajas.

Los Herero mantienen un claro sentimiento de agravio por considerarse las principales víctimas del proceso de desposesión colonial, mientras ahora legalmente tienen el mismo derecho a recibir tierras que grupos que nunca fueron expropiados, y manifiestan la percepción de que el gobierno favorece a los solicitantes Owambo. Esta demanda de reparación de los abusos coloniales no impide que a menudo reproduzcan visiones que marginalizan a los San. Los omitandu o poemas elegíacos Herero recuerdan la historia de lugares ligados con el pasado de la comunidad, y aunque reconocen que los San o los Damara se 
encontraban ya en algunas de las tierras que los Herero ocuparon, esto no implica una aceptación de un derecho de estos grupos sobre los terrenos (Hoffmann, 2005). De hecho, dentro de las ACs parece que es la zona este de la antigua Hereroland donde las condiciones de vida de los San son peores, por la tradición de malas relaciones entre ellos y los Herero locales, y por el aumento de hasta un $40 \%$ en el número de residentes San a causa de las expulsiones de trabajadores de las granjas comerciales. En el Omaheke, muchos Herero les siguen considerando "primitivos" o "salvajes" (Suzman, 2001; Sylvain, 1999).

En el Norte, los Owambo a menudo les consideran "gandules", sucios, incapaces de realizar correctamente tareas agrícolas o ganaderas, y pedigüeños. Con frecuencia los San son mal atendidos o mal tratados cuando deben acudir a oficinas del gobierno o a hospitales (Widlok, 1999). En 2005, WIMSA calculaba que había unas 518 familias San en la región de Ohangwena, comprendiendo a unas 2.000 personas. Muchos viven en Reasentamientos del gobierno, y la mayor parte se dedican a trabajar para sus vecinos Owambo, tanto en tareas agrícolas, como buscando agua y leña para ellos, o como trabajadores domésticos. A cambio reciben comida y tombo (cerveza de millo fermentado). Muy pocos San disponen de tierra propia, una situación que se ve agravada por el hecho de que no disponen de Autoridades Tradicionales reconocidas y, por tanto, de jefes San que tengan derecho a atribuir parcelas a personas de su comunidad. Aunque en los Reasentamientos han surgido algunos líderes San, su papel se limita a la resolución de conflictos internos y no son reconocidos ni respetados por los Owambo (Berger \& Mazive, 2002; Pakleppa, 2005). En 2005, los Coordinadores del MLR en los Reasentamientos eran Owambo, y en algunos casos los beneficiarios se quejaban de que les obligaban a trabajar para ellos (Berger \& Mazive, 2002; Pakleppa, 2005; Tapia, 2005). Los oficiales del MLR culpan del fracaso de los proyectos a los San, a quienes acusan de irresponsables, de no pensar en el día de mañana, y de vender los alimentos que les proporciona el MLR a cambio de tombo (Tapia, 2005). Por su lado, los San de Ekoka se quejan de que el jefe Owambo de la zona otorga parcelas de tierra dentro del Reasentamiento a Owambos (Dirkx \& Ayisa, 2007).

\section{Tsumkwe}

Los San sólo disponen de unas tierras que se consideren oficialmente como suyas, y donde se les han reconocido Autoridades Tradicionales y el derecho a gestionar los recursos naturales: el distrito de Tsumkwe, la antigua Bushmanland, en la región de Otjozondjupa. Esa zona, es una "sabana boscosa y arbustiva semiárida”, con muy poca agua, dependiente de la lluvia y de pozos excavados para 
explotar fuentes de agua subterráneas. La lluvia es irregular y el suelo sufre un nivel "extremadamente elevado de evaporación". Las posibilidades de practicar la agricultura y la ganadería eran muy marginales. El territorio había sido ocupado desde siempre por pequeñas comunidades San que se dedicaban básicamente a la caza y la recolección, y habían quedado muy al margen de la acción de alemanes y sudafricanos (Biesele \& Hitchcock, 2011; Botelle \& Rohde, 1995; Gordon, 1992; Hohmann, 2003; NACSO, 2012). En los años 1970, los sudafricanos establecieron bases militares en Bushmanland y trasladaron a la región a unos 4.000 San provenientes de Angola y Kavango. Tanto ellos como los Ju/'hoansi locales se alistaron en el ejército sudafricano por el salario relativamente elevado que se les ofrecía, por coerción, y por el éxito de la propaganda sudafricana que les anunciaba que una victoria de la Swapo significaría que perderían sus tierras a manos de los Owambo. Cuando la guerra terminó, unos 2.000 de los San de Angola y Kavango se fueron con los sudafricanos, dejando en Bushmanland a aproximadamente $2.100 \mathrm{Ju}$ /'hoan en la zona central y oriental y a unos 1.900 Mpungu y Vasekele San en la parte occidental (Botelle \& Rohde, 1995; Gordon, 1992).

Con el apoyo de activistas y antropólogos, los Ju/'hoan se organizaron en asociaciones y cooperativas que cristalizaron en la Nyae Nyae Development Foundation of Namibia (NNDFN) en 1991. Estas organizaciones se opusieron firmemente a los intentos de la administración sudafricana y de algunos elementos del nuevo gobierno independiente para convertir Eastern Bushmanland en una Reserva de Caza, y defendían un modelo de conservación comunitaria que asegurase el control de la tierra y los recursos naturales por parte de los Ju/'hoan, con el ecoturismo como una fuente de ingresos cada vez más importante en el marco de una economía mixta (Biesele \& Hitchcock, 2011).

Este objetivo fue facilitado por la Nature Conservation Amendment Act de 1996, que permitió la creación de Communal Conservancies, las cuales reciben el derecho de gestión de la fauna en su área de jurisdicción, la potestad exclusiva de autorizar concesiones turísticas y una cuota anual de animales para cazar, fijada por el gobierno (Boden, 2003; Hohmann, 2003; Rousset, 2003).

En 1998 la zona del East Tsumkwe District se convirtió en la Nyae Nyae Conservancy, la primera en registrarse en Namibia. En Western Bushmanland (West Tsumkwe), grupos de pastores Kwangali habían llevado su ganado a pastar en las tierras hasta entonces reservadas a los San, alegando que la Constitución permite a cualquier namibio residir donde desee (Suzman, 2001). En 1999 esta actitud provocó conflictos con los grupos San residentes. Para asegurarse el acceso y control de la tierra en West Tsumkwe, las comunidades locales establecieron una nueva Conservancy, Nła Jaqna, que fue reconocida en 2003, y en cuya 
constitución se dice que los miembros pueden ser personas residentes y con familiares en la zona, o que han recibido permiso de la Autoridad Tradicional o del Comité de la Conservancy para instalarse allí: de esta manera, la Conservancy está abierta a miembros no San, pero la gran mayoría lo son, y quien desee participar/ residir en la misma debe recibir permiso del Comité (Harring \& Odendaal, 2006; Hohmann, 2003; WIMSA, 2006-2007).

Pero ni aún con el reconocimiento oficial de las Conservancies, las tierras de los San en Tsumkwe están totalmente protegidas. Recientemente, el gobierno ha iniciado la planificación de un proyecto para instalar en la parte norte de $\mathrm{N} \neq \mathrm{a}$ Jaqna a pequeños granjeros comerciales, por el cual se espera que reciban parcelas individuales de 1.500 hectáreas cada uno y se dediquen a la ganadería comercial. La comunidad San local, con el apoyo de WIMSA, es muy reticente ante este plan y reclama ser consultada, que se garantice que los posibles beneficios serán para los San, y que se compruebe que los ingresos de estas parcelas serán mayores que los de la Conservancy (Harring \& Odendaal, 2006; WIMSA, 2006-2007). En proyectos como este, el gobierno muestra la permanencia de la idea de que amplias áreas de tierra de los San están "libres" o infrautilizadas y serían más provechosas bajo un régimen de agricultura comercial (y aunque no se diga oficialmente, desarrollado por comunidades más "avanzadas"). Desde los últimos años del régimen colonial, buena parte de las élites africanas asumieron la idea de que las propiedades individuales dedicadas a la agricultura comercial son más eficientes y que los vallados ayudan a mejorar las producciones. El gobierno independiente, además, no desea enfrentarse a las élites locales - con las que a menudo tiene lazos - y menos aún para defender a los San (Cox, 1998; Tapia, 2005).

Las cosas no están mucho mejor en Nyae Nyae. En abril de 2009 diversas familias Herero procedentes de Gam rompieron los vallados de la Conservancy y se introdujeron en la misma, junto con centenares de cabezas de ganado. Las autoridades arrestaron a algunos pastores, que salieron libres bajo una fianza mínima (Maletsky, 2009; Shejavali, 2009). Tanto la Conservancy como el Chief Tsamkxao Oma han solicitado al gobierno que actúe con más contundencia contra los invasores, y se han quejado de la poca celeridad con que han reaccionado las autoridades, así como de la ausencia de declaraciones oficiales claras contra los pastores Herero.

\section{Caprivi}

En la zona occidental de la franja de Caprivi (Western Caprivi), recientemente convertida en el Bwabwata National Park, las disputas entre los Khwe San locales y las poblaciones de pastores y agricultores Mbukushu que empezaron a instalarse 
en la zona en el siglo XVII han sido constantes en las últimas décadas (Rousset, 2003). De nuevo se ha combinado la historia de subordinación de ciertos grupos Khwe a los recién llegados, para los cuales trabajaban como pastores o domésticos, con la politización de los años de la guerrilla y la post-independencia. Existe la sensación entre los Khwe que el gobierno les discrimina frente a los Mbukushu porque ellos se alistaron en el ejército sudafricano y colaboraron con la guerrilla angoleña de la UNITA, y porque se supone que son votantes de partidos de la oposición (Pakleppa \& Kwononoka, 2003; Rousset, 2003).

Aunque en 1987 los Khwe eligieron un jefe, Kipi George, ni él ni ninguna otra autoridad Khwe han sido reconocidas oficialmente por el gobierno. En cambio, sí que se ha reconocido al jefe de los Mbukushu, Erwin Mlambo. Siguiendo la tradición histórica de los Mbukushu, el gobierno ha considerado que los Khwe eran en realidad un grupo subordinado a los Mbukushu y que debían quedar, por tanto, bajo la jurisdicción de Mlambo. Seguramente no es ajeno a esta decisión el hecho de que los Mbukushu sean considerados normalmente votantes de la Swapo y que Mlambo fuera un antiguo maestro de cuadros de la Swapo en el exilio (Boden, 2003; Rousset, 2003). Kipi George falleció en 2000 y los Khwe escogieron a su sustituto, el Chief Thaddeus Chedau, que tampoco ha sido reconocido por el gobierno. Actualmente, en el Western Caprivi hay unos 4.000 habitantes, de los cuales el 90\% son Khwe San y el 10\% Mbukushu (Rousset, 2003).

Los Khwe son mal vistos o menospreciados por los Mbukushu. Al ser una sociedad descentralizada y sin jefes que tuvieran la misma autoridad de los jefes Mbukushu, se les considera "desorganizados", y se exagera la historia de subordinación de los grupos Khwe a los Mbukushu. Como sus campos de cultivo son más pequeños que los de sus vecinos se les acusa de "gandules". Su lengua, el Khwekhwedam, no está presente en la escuela y la radio, a diferencia del Thimbukushu (Rousset, 2003).

El no reconocimiento de su Autoridad Tradicional coloca a los Khwe en una posición de continua desventaja: como no se les consulta a la hora de realizar proyectos de desarrollo en su región, no pueden aplicar sus leyes tradicionales ni acudir al jefe para los casos que juzgan los tribunales tradicionales deben dirigirse a los jefes Mbuskushu — al igual que cuando necesitan una parcela de tierra (Orth, 2003; Rousset, 2003). Los Khwe se lamentan también de discriminaciones a favor de los Mbukushu incluso en temas como la distribución de la ayuda en los años de sequía (WIMSA, comunicación personal, 23 febrero 2009). 


\section{Conclusión: ¿Una herencia colonial?}

En este artículo hemos intentado mostrar las características de la visión colonial — y la clasificación social y política correspondiente — sobre los San de Namibia. Y también resaltar como esta clasificación y las ideas que la sustentan se han prolongado de forma significativa después de la independencia del país. Las visiones que los europeos desarrollaron sobre los San a lo largo de siglos han influido en la forma como los namibios han construido su imaginario sobre los San tanto en el período colonial como en el postcolonial. Ahora bien, esta construcción colonial y su traspaso a épocas contemporáneas no explican toda la historia.

También hemos visto como, por un lado, la "visión" colonial distaba mucho de ser única y permanente. A lo largo del tiempo sectores diversos de las poblaciones y administraciones europeas han ido modificando su percepción de los San, alternando desde la negatividad más extrema hasta visiones más benévolas y/o paternalistas. Por otro lado, los africanos no se han limitado a asumir las opiniones de los europeos. A lo largo de la historia, ellos mismos han elaborado y adoptado/adaptado discursos y visiones que infravaloraban y discriminaban a los San. Ya en época pre-colonial abundaban las tradiciones que contemplaban a los Bosquimanos como una cultura sometida, servil o menos "desarrollada". Los grupos agropastorales consideraban la caza-recolección como una forma de vida "primitiva" comparada con la suya, y el uso de la tierra para estas actividades a menudo se equiparaba, de hecho, a un "no-uso". La caza y la recolección, por sí solas, no otorgaban legitimidad al control o propiedad de los territorios por parte de los San. La extrema movilidad de los grupos Bosquimanos, su descentralización y la ausencia de autoridades políticas reconocibles para los Bantu, reforzaban la percepción de los San como una sociedad poco desarrollada y sin control efectivo de sus tierras.

Estas ideas, como hemos visto, se intensificaron durante el período colonial, tanto por la extensión de las concepciones socioeconómicas y culturales occidentales entre los africanos como por el aumento de la subordinación de los San hacia los Bantu en las reducidas Reservas demarcadas por los gobiernos coloniales. Finalmente, tras la independencia, los discursos modernizadores defendidos por las élites políticas nacionalistas, el proyecto de Estado-nación uniformizador, y los intereses económicos de las diversas comunidades namibias - como la voluntad de conservar/extender las tierras bajo control de cada grupo, y la expansión de la agricultura comercial — se sumaron a la herencia histórica de relaciones de dominación para contribuir a la continuidad de una visión que alterna la superioridad cultural con el paternalismo y la voluntad de 
asimilación de los San. Como en muchos otros aspectos de la historia africana, no basta con invocar el pasado colonial para comprender un presente de injusticia o desigualdad.

Recebido em 23/06/2015.

Aceito em 02/08/2015.

Eduard Gargallo é investigador pós-doutoral no Instituto Universitário de Lisboa (ISCTE-IUL), Centro de Estudos Internacionais, Lisboa, Portugal. Atualmente realiza uma investigação sobre as políticas de terras e a conservação comunitária dos recursos naturais em Namíbia e Moçambique. Também foi diretor de Projetos do Centre d'Estudis Africans em Barcelona. Publicou artigos em revistas como Journal of Southern African Studies, Journal of Namibian Studies, Politique Africaine e Journal of Contemporary African Studies. Contato: egargallo@ yahoo.com

\section{Notas}

* Agradezco a la Fundação para a Ciência e a Tecnologia (FCT) de Portugal la concesión de una beca postdoctoral (Bolsa SFRH/BPD/68733/2010) que me ha permitido llevar a cabo la investigación necesaria para este artículo. Investigaciones previas en Namibia, que también se reflejan aquí, fueron posibles gracias a una beca de investigación de la Agencia Española de Cooperación Internacional al Desarrollo (AECID) en 2007-2009. También doy las gracias al Centro de Estudos Internacionais - Instituto Universitário de Lisboa (ISCTE-IUL) por las facilidades ofrecidas para mi trabajo como investigador en la institución. Finalmente, agradezco al profesor Wilson Trajano Filho sus comentarios a una versión previa de este artículo. 
1. La denominación Bushmen, extendida durante el período colonial para describir a estas poblaciones, fue adquiriendo un carácter despectivo y ha sido sustituida actualmente por el término San. Algunas comunidades y antropólogos, sin embargo, defienden el uso de Bushmen como válido. Nosotros hemos optado por combinar San y Bosquimano, la versión española de Bushmen, que no tiene carácter peyorativo.

2. Khoikhoi hace referencia a los diversos grupos llamados "Hotentotes" durante el período colonial. La denominación colonial es claramente despectiva y se ha evitado su uso.

3. Los Oorlam-Nama eran unas poblaciones Khoikhoi y mestizas provenientes de la Colonia del Cabo, donde habían adoptado la lengua afrikaans y las costumbres militares de los Afrikaners (como el Kommando), y que se establecieron y expandieron por el Sur y Centro de Namibia.

\section{Referências bibliográficas}

ADAMS, Fiona; WERNER, Wolfgang \& VALE, Peter. 1990. The land issue in Namibia: an inquiry. Windhoek: Namibian Institute for Social and Economic Research. University of Namibia.

BARNARD, Alan. 1992. Hunters and herders of Southern Africa: a comparative ethnography of the Khoisan peoples. Cambridge: Cambridge University Press.

BERGER, Dhyani J. \& MAZIVE, Elke Z. 2002. New horizons for the San: participatory action research with San communities in Northern Namibia. Windhoek: Unesco.

BIESELE, Megan \& HITCHCOCK, Robert K. 2011. The Jul'hoan San of Nyae Nyae and Namibian independence: development, democracy, and indigenous voices in Southern Africa. New York and Oxford: Berghahn Books.

BODEN, Gertrud. 2003. "Caught in the middle': impacts of state decisions and armed conflicts on Khwe economy and ethnicity in West Caprivi between 1998 and 2002". In: Thekla Hohmann (ed.). San and the state: contesting land, development, identity and representation. Köln: Rüdiger Köppe. pp. 161-204.

BOTELLE, Andy \& ROHDE, Rick. 1995. Those who live on the land: a socio-economic baseline survey for land use planning in the communal areas of Eastern Otjozondjupa. Windhoek: Ministry of Lands, Resettlement and Rehabilitation.

CHIDESTER, David. 1996. "Mutilating meaning: European interpretations of Khoisan languages of the body". In: Pippa Skotnes (ed.). MISCAST: negotiating the presence of the Bushmen. Ciudad del Cabo: University of Cape Town Press. pp. 24-38. 
COX, Jonathan. 1998. "The research context". In: Jonathan Cox; Carol Kerven; Wolfgang Werner \& Roy Behnke. The privatisation of rangeland resources in Namibia: enclosure in Eastern Oshikoto. London: Overseas Development Institute. pp. 8-17.

DIECKMANN, Ute. 2007. Hail/om in the Etosha region: a history of colonial settlement, ethnicity and nature conservation. Basilea: Basler Afrika Bibliographien.

DIRKX, Eric-Jan \& AYISA, Kofi. 2007. "Feasability study for Onamatadiva, Eendobe, Oshanashiwa and Ekoka resettlement projects". In: Desert Research Foundation of Namibia. PRA findings: final report. Windhoek: Desert Research Foundation of Namibia (DRFN) and Acacia Grassroots Development Network.

DESERT RESEARCH FOUNDATION OF NAMIBIA (DRFN). 2006a. "Drimiopsis San resettlement project. Omaheke region. Site report". Windhoek: DRFN - Fundación CEAR.

. 2006b. "PRA/Situational analysis: site report for Skoonheid resettlement farm. Omaheke Region”. Windhoek: DRFN - Fundación CEAR.

ELPHICK, Richard. 1977. Kraal and castle: Khoikhoi and the founding of White South Africa. New Haven \& London: Yale University Press.

GORDON, R. 1992. The Bushman myth: the making of a Namibian underclass. Boulder: Westview Press.

. 1997. Picturing Bushmen: the Denver Africa Expedition of 1925. Athens: Ohio University Press.

. 2003. "Gathering the hunters: Bushmen in German (colonial) anthropology". In: H. Glenn Penny \& Matti Bunzl (eds.). Worldy provincialism: German anthropology in the age of empire. Ann Arbor: University of Michigan Press. pp. 256-282.

GRAEFE, Olivier \& PEYROUX, Élisabeth. 1999. "La décentralisation à l'épreuve des faits: l'exemple d'Oshakati, capitale économique de l'ancien Owamboland". In: Ingolf Diener \& Olivier Graefe (dirs.). La Namibie contemporaine: les premiers jalons d'une société post-apartheid. Paris: Karthala-IFRA. pp. 279-292.

GUENTHER, Mathias. 1999. Tricksters and trancers: Bushman religion and society. Bloomington: Indiana University Press.

HAHN, Carl H. L.; FOURIE, Louis \& VEDDER, Heinrich. 1928. The native tribes of South West Africa. Ciudad del Cabo: Cape Times. 
HARRING, Sidney. 1998. "Inconsistencies in the constitution of Namibia regarding the land question”. In: Franka M. d'Engelbronner-Kolff; Manfred O. Hinz \& J. L. Sindano (eds.). Traditional authority and democracy in Southern Africa. Windhoek: Centre for Applied Social Studies. University of Namibia \& New Namibia Books. pp. 155-182.

HARRING, Sidney \& ODENDAAL, Willem. 2006. "Our land they took": San Land rights under threat in Namibia. Windhoek: Legal Assistance Centre.

HINZ, Manfred O. 1998. "Communal land, natural resources and traditional authority". In: Franka M. d'Engelbronner-Kolff; Manfred O. Hinz \& J. L. Sindano (eds.). Traditional authority and democracy in Southern Africa. Windhoek: Centre for Applied Social Studies. University of Namibia \& New Namibia Books. pp. 183-227.

HOFFMANN, A. G. 2005. "Since the Germans came it rains less": landscape and identities of Herero communities in Namibia. Tesis doctoral, University of Amsterdam.

HOHMANN, Thekla. 2003. “We are looking for life. We are looking for the conservancy'. Namibian conservancies, nature conservation and rural development: the Nła-Jaqna conservancy”. In: . (ed.). San and the State: contesting land, development, identity and representation. Köln: Rüdiger Köppe. pp. 205-254.

KEULDER, Christopher. 1998. "Traditional leaders and rural development". In: Franka M. d'Engelbronner-Kolff; Manfred O. Hinz \& J. L. Sindano (eds.). Traditional authority and democracy in Southern Africa. Windhoek: Centre for Applied Social Studies. University of Namibia \& New Namibia Books. pp. 289-322.

. 2000. "Traditional leaders". In: . (ed.). State, society and democracy: a reader in Namibian politics. Windhoek: Gamsberg Macmillan. pp. 150-170.

KÖSSLER, Reinhart. 2005. In search of survival and dignity: two traditional communities in southern Namibia under South African rule. Windhoek: Gamsberg Macmillan.

KREIKE, Emmanuel. 1996. "Recreating Eden: agro-ecological change, food security and environmental diversity in Southern Angola and Northern Namibia, 1890-1960". Tesis doctoral, Yale University.

MALETSKY, Christof. 2009. "Herero farmers invade San area". The Namibian, 6 de mayo.

MARSHALL THOMAS, Elizabeth. 1959. The harmless people. London: Secker \& Warburg.

McKITTRICK, M. 2008. "Landscapes of power: ownership and identity on the middle Kavango River, Namibia”. Journal of Southern African Studies, 34(4):785-802. 
MINISTRY OF LANDS AND RESETTLEMENT (MLR). 2005a. Background research work and findings of the permanent technical team studies. Windhoek. .2005b. Strategic options and action plan for land reform in Namibia. Windhoek.

MINISTRY OF LANDS, RESETTLEMENT AND REHABILITATION (MLRR). 1997. National Resettlement Policy. Windhoek. 1998. National land policy. Windhoek.

MOSOTHO, Kesentseng \& TSIU, Mapaseka J. 2008. Assessment of land use on the resettlement farms Lievenberg, Drimiopsis and Du Plessis. Windhoek: Namibia Institute for Democracy. Analyses and Views, 5 (4).

Namibian Association of CBNRM Support Organisations (NACSO). 2012. Living with wildlife - the story of Nyae Nyae conservancy. Windhoek.

ODENDAAL, Willem \& TJIRAMBA, Shadrack. 2005. Our land we farm: an analysis of the Namibian commercial land reform process. Windhoek: Legal Assistance Centre.

ORTH, Ina. 2003. "Identity as dissociation: the Khwe's struggle for land in West Caprivi”. In: Thekla Hohmann (ed.). San and the state: contesting land, development identity and representation. Köln: Rüdiger Köppe pp. 121-159.

PAKLEPPA, Richard. 2005. Report on enquiry into the socio-economic conditions and needs of some San communities in the Ohangwena and Otjikoto regions of Northern Namibia. Windhoek: WIMSA.

PAKLEPPA, Richard \& KWONONOKA, Americo. 2003. Onde os primeiros são os últimos: a luta pela supervivência das comunidades San no sul de Angola. Windhoek: Trócaire Angola-WIMSA-OCADEC.

PANKHURST, Donna. 1996. A resolvable conflict? The politics of land in Namibia. Peace Research Report no 36. Department of Peace Studies. University of Bradford.

PEACOCK, S. 1999. "Africa meets the great Farini”. In: Bernth Lindfors (ed.). Africans on stage: studies on ethnological show business. Bloomington: Indiana University Press. pp. 81-106.

PENN, N. 1996. “'Fated to perish': the destruction of the Cape San”. In: Pippa Skotnes (ed.). MISCAST: negotiating the presence of the Bushmen. Ciudad del Cabo: University of Cape Town Press. pp. 81-91.

. 2005. The forgotten frontier: colonist and Khoisan on the Cape's Northern frontier in the 18th century. Ciudad del Cabo: Double Storey Books. 
PISANI, André du. 2000. "State and society under South African rule". In: Christopher Keulder (ed.). State, society and democracy: a reader in Namibian politics. Windhoek: Gamsberg Macmillan. pp. 49-76.

ROBINS, Steven. 2000. "Land struggles and the politics and ethics of representing 'Bushman' history and identity”. Kronos, 26:58-75.

ROUSSET, Karine. 2003. To be Khwe means to suffer: local dynamics, imbalances and contestations in the Caprivi Game Park. Tesis de Licenciatura, University of Cape Town.

SHEJAVALI, Nangula. 2009. "Gam farmers to experience consequences of land invasion”. The Namibian, May 8.

SILVESTER, Jeremy. 1993. Black pastoralists, white farmers: the dynamics of land disposession and labour recruitment in Southern Namibia, 1915-1955. Tesis doctoral, University of London.

SILVESTER, Jeremy \& GEWALD, Jan-Bart (eds.). 2003. Words cannot be found: German colonial rule in Namibia: an annotated reprint of the 1918 Blue Book. Leiden: Brill.

SMITH, Andy; MALHERBE, Candy; GUENTHER, Mat \& BERENS, Penny. 2000. The Bushmen of Southern Africa: a foraging society in transition. Ciudad del Cabo \& Athens: David Philip \& Ohio University Press.

SUZMAN, James. 2000. "Things from the bush": a contemporary history of the Omaheke Bushmen. Basilea: P. Schlettwein Publishing.

. 2001. An assessment of the status of the San in Namibia. Windhoek: Legal Assistance Centre.

SYLVAIN, Renée. 1999. "We work to have life": Jul' hoan women, work and survival in the Omaheke region. Tesis doctoral. University of Toronto.

TAPIA, Carlos. 2005. La reforma agraria en Namibia: ¿una alternativa de desarrollo?. Florencia: Instituto Agronomico per l'Oltremare. Società Editrice Fiorentina.

TÖTEMEYER, G. 2000. "Decentralisation and state-building at the local level". In: Christopher Keulder (ed.). State, society and democracy: a reader in Namibian politics. Windhoek: Gamsberg Macmillan. pp. 108-149.

VAN DER POST, Laurence. 1958. The lost world of the Kalahari. Penguin: Harmondsworth.

WERNER, Wolfgang. 1998. "No one will become rich". In: Economy and society in the Herero reserves in Namibia, 1915-1946. Basilea: P.Schlettwein Publishing. 
. 2004. "Promoting development among farm workers: some options for Namibia". In: J. Hunter (ed.). Who should own the land? Analyses and views on land reform and the land question in Namibia and Southern Africa. Windhoek: Konrad-AdenauerStiftung \& Namibia Institute for Democracy. pp. 19-45.

WIDLOK, T. 1999. Living on Mangetti: "Bushman" autonomy and Namibian independence. Oxford: Oxford University Press.

. 2003. "The needy, the greedy and the state: dividing Hai//om Landin the Oshikoto Region”. In: Thekla Hohmann (ed.). San and the State: contesting land, development, identity and representation. Köln: Rüdiger Köppe. pp. 87-119.

WIMSA. 2006-2007. Report of activities: April 2006-March 2007. Windhoek: Working Group on Indigenous Minorities in Southern Africa.

.2007-2008. Report of activities: 2007-2008. Windhoek: Working Group on

Indigenous Minorities in Southern Africa. 


\section{Resumen}

El presente artículo intenta analizar la importancia de la visión colonial sobre las poblaciones San de Namibia y su impacto en las políticas desarrolladas por la administración alemana y sudafricana del territorio. Asimismo, muestra como estas visiones fueron en buena parte incorporadas y adaptadas por las demás comunidades africanas y como han influido en las políticas impulsadas por la Namibia independiente. El análisis se centra especialmente en las políticas de tierras de los gobiernos coloniales y postcoloniales, que han reducido al mínimo las áreas reconocidas como pertenecientes a los San. El artículo muestra como las visiones coloniales, a menudo negativas y estereotipadas sobre los San, no fueron homogéneas ni inamovibles, y como fueron adoptadas por los africanos de Namibia y han perdurado hasta la actualidad.

Palabras clave: comunidades San; Namibia; visión colonial; tierra.

\section{Abstract}

This article analyses the colonial visions on San populations in Namibia and their impact on the policies implemented by German and South African Administrations. It also seeks to show how these visions were partly assimilated by other African communities and they have influenced some Namibian policies after independence. The analysis focuses especially on land policies implemented by both colonial and postcolonial governments, which have recognised only very small areas as belonging to the San. The article shows how colonial visions, often negative and full of stereotypes about the San, were neither homogeneous nor fixed, and how they were adopted by Namibian Africans and have lasted until the present.

Keywords: San communities; Namibia; colonial visions; land. 